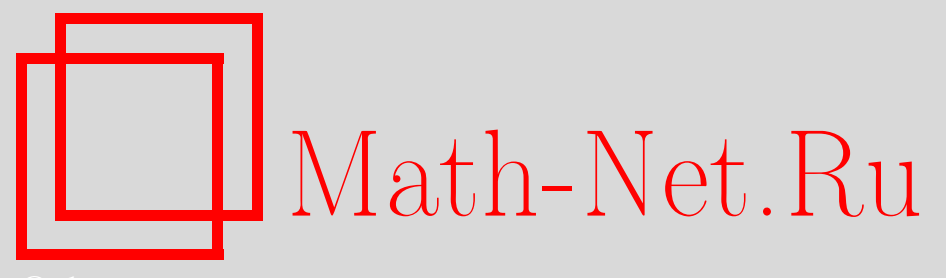

С. Дворянинов, Парадокс Толи Втулкина, Квант, 2020, номер 5, 41-45

DOI: https://doi.org/10.4213/kvant20200505

Использование Общероссийского математического портала Math-Net.Ru подразумевает, что вы прочитали и согласны с пользовательским соглашением http://www.mathnet.ru/rus/agreement

Параметры загрузки:

IP : 54.80 .97 .219

26 апреля 2023 г., $17: 42: 59$

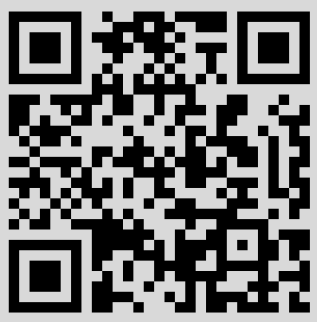




\section{Парадокс Толи Втулкина}

\section{С.ДВОРЯНИНОВ}

\section{Часть I}

Вниманию читателей предлагается научно-фантастический рассказ для будущих физиков-теоретиков.

Уже много лет прошло с тех пор, как на нашем школьном физическом кружке Толя Втулкин демонстрировал изобретенную им экспериментальную установку. А кажется, это было вчера...

Конструкция очень простая. Главная ее часть - тонкая стеклянная трубка, но не прямая, а изогнутая в виде полуокружности радиусом $R$. Есть еще металлический шарик 1 , масса которого $m$. Он очень маленький и может свободно двигаться внутри трубки. Трубка закреплена на штативе в вертикальной плоскости в форме буквы C (рис.1). Ее верхний конец $B$ и нижний $H$ располагаются точно на одной вертикали. Когда мы пытались поместить шарик в точку $B$, то это нам никак не удавалось. И ясно, почему: это положение шарика неустойчиво. А еще к точке $B$ была привязана тонкая нить длиной $2 R$. К этой нити можно было привязывать другие шарики, которых были три. Массы $M$ у

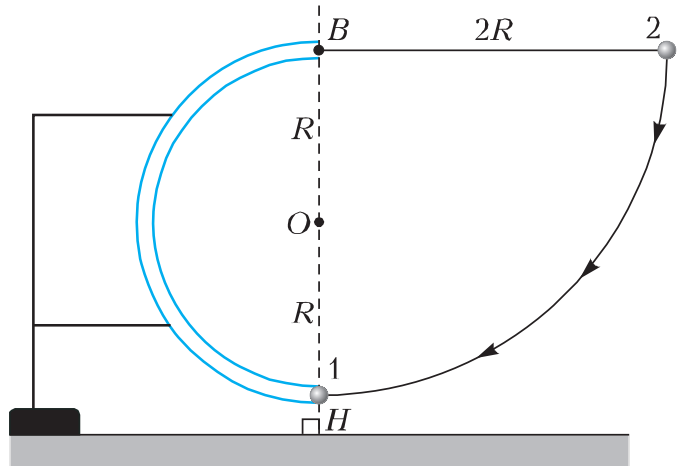

РИс. 1

DOI: https://doi.org/10.4213/kvant20200505 них были разные, а диаметры - чуть больше диаметра трубки.

Сначала Толик поместил наш шарик 1 в точку $H$, а к нити привязал легкий шарик 2 , масса которого $M$ существенно меньше массы $m$. Затем он отвел нить в сторону и натянул ее. В результате нить расположилась вдоль горизонтали. Это, можно сказать, была стартовая позиция.

- Что будет происходить дальше, если я выпущу свой шарик 2 из рук? - спросил Толик.

Ответ был всем очевиден. Шарик в руках у Толика обладает потенциальной энергией $M g \cdot 2 R$. Он, двигаясь вниз как маятник, столкнется с шариком 1 и передаст ему часть своей кинетической энергии. Шарик 1, обладая некоторой энергией, начнет двигаться по трубке вверх, но, не дотянув даже до уровня точки $O$, на миг остановится, потом начнет скатываться вниз и выкатится из трубки. Так оно и оказалось на опыте.

Во второй раз Толик привязал к нити тяжелый шарик 2 , масса $M$ которого много больше $m$. И эта ситуация была понятна. Шарик 1 после удара по нему шарика 2 получил такую порцию энергии, что смог подняться по трубке до точки $B$, причем с ненулевой скоростью. И действительно, шарик 1 вылетал из трубки, как из пушки.

После этих простых опытов некоторые стали было терять всякий интерес к Толиной установке.

- Подождите, не уходите, сейчас будет самое интересное, - воскликнул Толик. Пожалуйста, посмотрите! - с этими словами он привязал к нити шарик 2 с массой $m$, отвел нить в сторону и натянул.

- Что теперь будет с шариком 1, который сейчас покоится в точке $H$, после того как я выпущу из рук свой шарик 2? А? Какие ваши предположения? - обратился ко всем Толик.

Дело это всем показалось нехитрым. Потенциальная энергия шарика 2 в руках у Толика равна $2 m g R$. После столкновения она переходит в энергию шарика 1 с массой $m$. Этой энергии ему в точности, тютелька в тютельку, хватит для того, чтобы по трубке подняться в точку $B$. 
Примерно таким был наш ответ на вопрос Толика.

- Так, хорошо. Вы говорите - подняться в точку $B$, т.е. оказаться в точке $B$ ? продолжал допытываться Толик.

- Да, именно так, оказаться в точке $B$, какие могут быть в этом сомнения? настаивали мы.

- А вот и нет! Смотрите! - торжествующе произнес Толик и выпустил из рук свой шарик 2 с массой $m$.

Его шарик 2, двигаясь на нити вниз, столкнулся с неподвижным шариком 1 и остановился. А бывший до этого неподвижным шарик 1 начал по трубке двигаться вверх, все выше и выше. Все происходило так, как мы и предполагали...

Здесь уместно вспомнить о хорошо известном опыте с подвешенными на нитях одинаковыми шариками. На рисунке 2 изображены пять одинаковых шариков,

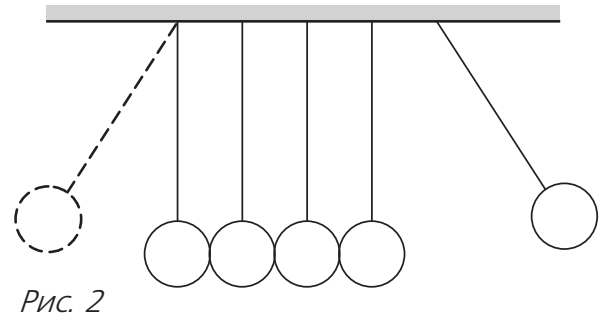

касающихся один другого и подвешенных на горизонтальной штанге. Если крайний правый шарик вывести из положения равновесия, отклонив его нить на некоторый угол от вертикали, и затем отпустить, то в результате серии ударов его импульс перейдет к крайнему левому шарику. Тот выйдет из положения равновесия, причем его нить отклонится от вертикали на тот же самый угол. Далее процесс будет повторяться. При этом три средних шарика будут оставаться (или казаться?) неподвижными.

...А дальше на нашем кружке началось непредвиденное. Шарик карабкался вверх, его скорость уменьшалась, он был все ближе и ближе к точке $B$, но...

Помните, как у М.Булгакова в романе «Мастер и Маргарита» говорится о погоне поэта Ивана Бездомного за Воландом и его свитой: «Сколько Иван ни прибавлял шагу, расстояние между преследуемым и им ничуть не сокращалось» .

Так и у нас: время шло, а шарик все катился и катился по трубке вверх, не останавливаясь. Нам даже стало казаться, что он неподвижен, столь мала была его скорость. Но взяв в руки лупу, мы смогли увидеть, что нет - шарик неустанно перемещался по трубке выше и выше. Картина была удивительной: со стороны казалось, что шарик стоит на месте, на дуге окружности, слева от точки $B$, но вниз не катится, словно нет никакой скатывающей силы.

Когда на следующий день мы пришли в класс, картина по-прежнему оставалась неизменной.

...Прошло много лет, а установка Толи Втулкина до сих пор стоит в школьном шкафу и неутомимый в своем движении шарик продолжает поражать всякого, увидевшего ее.

Сразу скажем, что разобраться с этим удивительным явлением не так-то просто. Но, попробуем.

\section{Часть II}

Герой этой части статьи (так же, как и ее первой части) - маятник. Рассказать о нем автора вдохновили замечательные статьи А.Мигдала и В.Арнольда, опубликованные в юбилейном номере «Кванта» (№1 за 2020 год).

Наш маятник - это тонкий невесомый стержень длиной $L$ с точечной массой $m$ на конце, который может, совершая полный оборот (сальто-мортале), вращаться в вертикальной плоскости (рис.3). Начнем мы с энергии маятника и запишем ее закон со- Рис. 3 хранения:

$$
\frac{m v^{2}(t)}{2}+m g h(t)=\text { const } .
$$

Здесь $v(t)$ - линейная скорость точечной массы $m, h=L-L \cos \varphi$ - высота подъема маятника от крайнего нижнего положения, $\varphi$ - угол отклонения от вертикали. Итак,

$$
\frac{m v^{2}(t)}{2}+m g(L-L \cos \varphi(t))=\text { const } \text {. }
$$

Сократив на массу $m$ и введя обозначение 


$$
\begin{gathered}
\frac{v^{2}(t)}{2 L g}=u^{2}(t), \text { уравнение (1) приведем к виду } \\
u^{2}=\cos \varphi+C,
\end{gathered}
$$

где величина $u$ с точностью до постоянного коэффициента есть линейная скорость, а $C$ параметр. На время забудем, что в уравнении (2) переменные $u$ и $\varphi$ зависят от времени, и построим график этого уравнения на плоскости с координатами $(u ; \varphi)$. Для этого рассмотрим вспомогательную функцию (точнее, семейство функций, зависящих от параметра $C$ )

$$
u=\sqrt{\cos \varphi+C} .
$$

Эта функция $2 \pi$-периодическая, поэтому сначала можно построить ее график на отрезке $-\pi \leq \varphi \leq \pi$, а потом периодически продолжить на всю прямую $-\infty<\varphi<\infty$. Затем к этому графику надо добавить график функции $u=-\sqrt{\cos \varphi+C}$. В итоге получится график уравнения (2).

На отрезке $-\pi \leq \varphi \leq 0$ функция $f(\varphi)=$ $=\cos \varphi+C$ возрастает, на отрезке $0 \leq \varphi \leq \pi$ убывает. Функция $\sqrt{f}$ возрастает в своей области определения, причем промежутки монотонности функции (3) совпадают с промежутками монотонности функции косинуса (разумеется, в своей области определения). А область определения функции (3) задается неравенством $\cos \varphi+C \geq 0$. Ясно, что эта область определения не пуста, если $C \geq-1$. Рассмотрим несколько возможных случаев.

1. Если $C=-1$, то получаем единственную точку $(0 ; 0)$ (точка 0 на рисунке 4 ). Этой точке соответствует неподвижный маятник, находящийся в крайнем нижнем устойчивом положении. При этом плоскость $(u ; \varphi)($ т.е. плоскость $(v ; \varphi)$,

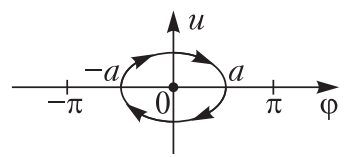

Pnc. 4 или $\left.\left(\varphi^{\prime} ; \varphi\right)\right)$ называется фазовой плоскостью маятника.

2. Если $C \in(-1 ; 1)$, то функция (3) определена на отрезке вида $[-a ; a] \subset[-\pi ; \pi]$, т.е на отрезке $[-\arccos (-C) ; \arccos (-C)]$ (см. рис.4). Изменению аргумента $\varphi$ на этом отрезке соответствуют колебания маятника. Двум точкам на плоскости $(u ; \varphi)$ с координатами $(-\arccos (-C) ; 0)$ и $(\arccos (-C) ; 0)$ соответствуют два крайних положения маят- ника - левое и правое. В этих точках пространства маятник «на мгновение замирает», т.е. его скорость становится равной нулю.

Из опыта известно, что из крайнего левого положения маятник попадает в крайнее правое и обратно за конечное время, которое называют периодом колебаний. На плоскости $(u ; \varphi)$ этим колебаниям соответствует замкнутая траектория. Касательная к этой траектории в крайних левой и правой точках вертикальна. Это следует из того, что производная функции $u=\sqrt{\cos \varphi+C}$ при $\varphi=$ $= \pm \arccos (-C)$ не существует (или обращается в бесконечность). Если теперь на графике (см. рис.4) поставить стрелки, соответствующие возрастанию времени $t$, то части графика превратятся в фазовые траектории.

В уравнении (3) вернемся к линейной скорости $v(t)$ :

$$
\frac{v(t)}{\sqrt{2 L g}}=\sqrt{\cos \varphi(t)+C} .
$$

Линейная скорость связана с угловой скоростью формулой $v=\omega L$, а переменная угловая скорость - это производная от угла отклонения маятника от вертикали, т.е. $\varphi^{\prime}(t)$, поэтому получаем уравнение

$$
\sqrt{\frac{L}{2 g}} \cdot \varphi^{\prime}=\sqrt{\cos \varphi+C},
$$

или

$$
\varphi^{\prime}(t)=K \sqrt{\cos \varphi(t)+C},
$$

где $K=\sqrt{\frac{2 g}{L}}$. Это - дифференциальное уравнение, которые связывает неизвестную функцию $\varphi(t)$ и ее производную.

В окрестности правой точки $(\arccos (-C) ; 0)$ (или $(a ; 0))$ функция $f(\varphi)=\cos \varphi+C \ll$ похожа» на линейную функцию $f(\varphi)=k(a-\varphi)$. Значит, на отрезке $[a-\varepsilon ; a]$ уравнение (4) можно заменить близким, но более простым дифференциальным

уравнением $\varphi^{\prime}=K \sqrt{k(a-\varphi)}, \quad$ или

$$
\varphi^{\prime}=p \sqrt{a-\varphi},
$$

где $p$ - некоторое положительное число.

Мы не требуем от наших читателей умения решать дифференциальные уравнения, достаточно лишь уметь дифференцировать. 
Можно проверить, что функция

$$
\varphi(t)=a-\frac{(p t-2 \sqrt{\varepsilon})^{2}}{4}
$$

удовлетворяет уравнению (5) и начальному условию $\varphi(0)=a-\varepsilon$. Эта функция равна $a$ при $t=\frac{2 \sqrt{\varepsilon}}{p}$. Другими словами, на временно́м отрезке $0 \leq t \leq \frac{2 \sqrt{\varepsilon}}{p}$ угол отклонения маятника от вертикали возрастает от $a-\varepsilon$ до наибольшего значения $a=\arccos (-C)$. Тем самым, мы рассмотрели математическую модель хорошо известного явления: при приближении маятника к крайним левому или крайнему правому положению (там, где скорость маятника равна нулю) его скорость монотонно убывает до нуля, но при этом в крайние положения маятник попадает за конечное время.

3. Рассмотрев два первых случая, мы узнали, как выглядят фазовые траектории уравнения (2) при каждом значении параметра $C$ из промежутка $[-1 ; 1)$ : это или точки, или овалы. А что будет, если $C \in(1 ;+\infty)$ ? Фазовые траектории для этого случая, пожалуй, самые простые (если не считать точек). Таким значениям $C$ соответствуют те значения механической энергии маятника, которые больше потенциальной энергии в самой верхней точке его пути. Другими словами, такой маятник, находясь на самом верху, обладает еще и кинетической энергией, т.е. ненулевой скоростью. Движение такого маятника - это не колебания туда-сюда, влевовправо, а постоянное вращение против или по часовой стрелке. При этом ясно, что его линейная скорость (как и угловая) меняется: при движении маятника вниз она нарастает, а после прохождения крайнего нижнего положения - уменьшается. Это хорошо видно на графике функции $u=\sqrt{\cos \varphi+C}$ (или на графике $u=-\sqrt{\cos \varphi+C}$ ) (рис.5). В верх-

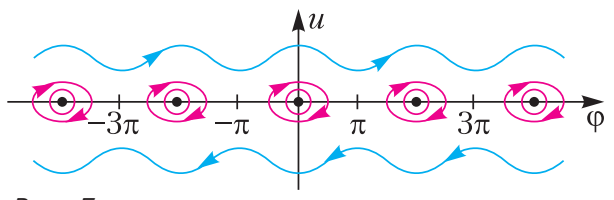

Рис. 5 ней полуплоскости стрелки на фазовых траекториях направлены вправо, в сторону возрастания $\varphi$, траектории описывают вращение маятника против часовой стрелки. В нижней полуплоскости стрелки на траектории направлены влево - маятник вращается по часовой стрелке.

4. Рассмотрим теперь функцию (3) при $C=1$ :

$$
u=\sqrt{\cos \varphi+1} .
$$

Она определена на всей прямой $-\infty<\varphi<+\infty$, неотрицательна и равна нулю при $\varphi=\pi+2 \pi n$ (рис.6). В этих точках график имеет излом

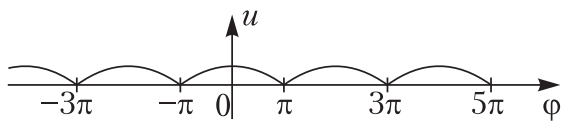

Рис. 6

(это следует из того, что здесь функция (6) имеет односторонние производные $\pm \sqrt{2}$ ). А какие фазовые траектории соответствуют графику этой функции? Сколько их?

На графике есть точки $(\pi+2 \pi n ; 0),\{n\}=Z$. Они соответствуют верхнему неустойчивому положению маятника. Каждая такая точка есть траектория динамической системы. Пусть при $t=0$ маятник находится в самой нижней точке, т.е. $\varphi=0$, и его скорость положительна. С течением времени угол $\varphi(t)$ увеличивается, скорость (и линейная, и угловая) уменьшается. Возникает вопрос: достигнет ли маятник самого верхнего положения и остановится ли он там? (В разных случаях ответ на этот вопрос разный. Об этом рассказывается, например, в статье С.Дворянинова, 3.Краутера и В.Протасова «Сколько времени длится причаливание?» в «Кванте» №11 за 2017 год.)

Рассмотрим функцию (6) на отрезке $\pi-\varepsilon \leq \varphi \leq \pi$ :

$$
u=\sqrt{2} \cdot \cos \frac{\varphi}{2} .
$$

Воспользуемся связью этой функции с линейной и угловой скоростями:

$$
u=\frac{v(t)}{\sqrt{2 L g}}=\frac{L \varphi^{\prime}(t)}{\sqrt{2 L g}}=\sqrt{\frac{L}{2 g}} \cdot \varphi^{\prime}(t)
$$

и получим следующее уравнение для угловой скорости $\varphi^{\prime}(t)$ :

$$
\varphi^{\prime}(t)=2 \sqrt{\frac{g}{L}} \cdot \cos \frac{\varphi}{2} .
$$


Будем теперь рассматривать новую функцию $\alpha(t)=\pi-\varphi(t)$, т.е. отклонение функции $\varphi(t)$ от $\pi$, при этом $0 \leq \alpha \leq \varepsilon$. Для новой функции имеем уравнение

$$
-\alpha^{\prime}(t)=2 \sqrt{\frac{g}{L}} \cdot \cos \left(\frac{\pi}{2}-\frac{\alpha}{2}\right),
$$

или

$$
\alpha^{\prime}(t)=-2 \sqrt{\frac{g}{L}} \cdot \sin \frac{\alpha}{2} .
$$

Хорошо известно, что синус малого аргумента приближенно равен самому аргументу, поэтому вместо уравнения (7) рассмотрим приближенное уравнение

$$
\beta^{\prime}(t)=-\sqrt{\frac{g}{L}} \cdot \beta .
$$

Так как на отрезке $0 \leq \alpha \leq \varepsilon$ справедливо неравенство $\sin \alpha \leq \alpha$, то функция $\beta(t)$ убывает быстрее функции $\alpha(t)$. Легко проверить, что решением последнего уравнения является функция

$$
\beta(t)=(\pi-\varepsilon) \cdot e^{-\sqrt{\frac{g}{L}} \cdot t},
$$

или

$$
\beta(t)=(\pi-\varepsilon) \cdot \exp \left(-\sqrt{\frac{g}{L}} \cdot t\right) .
$$

Эта функция убывает до нуля бесконечно долго. Поэтому функция $\alpha(t)$, убывающая еще медленнее, тем более будет убывать до нуля бесконечно долго! Это означает, что движение фазовой точки по сепаратрисе так называется кривая, задаваемая уравнением (6) на интервале $(-\pi ; \pi)$, - длится бесконечно долго. Сепаратриса - это дуга кривой конечной длины без концевых точек. На фазовой плоскости сепаратрис бесконечно много.

Все фазовые траектории маятника показаны на рисунке 7. Они делятся на четыре вида: точки, овалы, сепаратрисы, бесконечные кривые (изображены разными цветами).

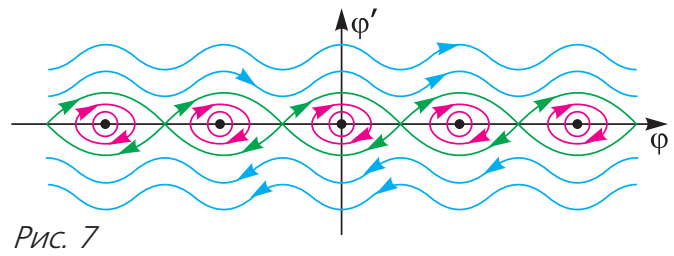

Заметим, что в установке Толи Втулкина (о ней рассказывается в первой части статьи) шарик никогда не останавливается именно потому, что соответствующая ему фазовая точка движется по сепаратрисе. В реальности, конечно, провести такой опыт не удастся - попасть на сепаратрису невозможно (вспомните знакомые вам выражения: сепаратный мир, сепаратные переговоры, сепаратор в подшипнике и т.п.).

В заключение - задача:

Невесомый стержень $\mathrm{OO}_{1}$ длиной $2 \mathrm{~L}$ может вращаться в вертикальной плоскости вокруг точки О (рис.8). С ним связан

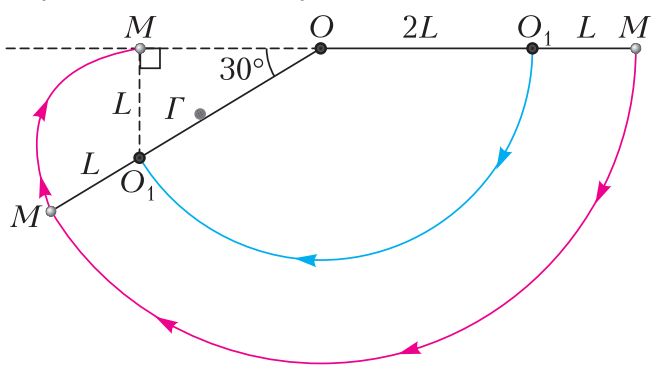

Pис. 8

шарниром невесомый стержень $O_{1} M$ длиной L, на конце которого закреплена точечная масса. Этот двухзвенныи маятник удерживают в горизонтальном положении, а затем отпускают без начальной скорости. На пути верхнего стержня маятника находится гвоздь Г. Луч ОГ образует угол $60^{\circ}$ с вертикалью, а длина отрезка ОГ меньше 2L. Докажите, что такой маятник никогда не вернется в исходное положение.

Ясно, что в тот момент, когда наш маятник, вращаясь вокруг точки $O$, «наткнется» на гвоздь, он превратится в маятник длиной $L$, вращающийся вокруг точки $O_{1}$. При этом запаса его кинетической энергии будет ровно столько, сколько требуется точке $M$ для подъема на начальную высоту. Это означает, что движению точки $M$ по окружности радиусом $L$ будет соответствовать движение фазовой точки по сепаратрисе. Но это движение длится бесконечно долго. Следовательно, укороченный маятник будет подниматься вверх бесконечно долго. Именно поэтому он никогда не вернется в исходное положение. 